\title{
Insulin and non-esterified fatty acid relations to alimentary lipaemia and plasma concentrations of postprandial triglyceride- rich lipoproteins in healthy middle-aged men
}

\author{
S. Boquist, A.Hamsten, F. Karpe, G. Ruotolo \\ Atherosclerosis Research Unit, King Gustaf V Research Institute, Department of Medicine, Karolinska Institute, \\ Karolinska Hospital, Stockholm, Sweden
}

\begin{abstract}
Aims/hypothesis. Enhanced and prolonged postprandial lipaemia is related to cardiovascular disease but how postprandial lipaemia is regulated is poorly known. We therefore determined the relations of fasting insulin concentrations to fasting and postprandial lipids, lipoproteins and non-esterified fatty acids in middle-aged men.

Methods. The subjects, 99 healthy 50-year-old men with an apolipoprotein E3/3 genotype, ate a mixed meal. The apolipoprotein B-48 and B-100 contents were determined in triglyceride-rich lipoproteins as a measure of chylomicron remnant and very low density lipoprotein particle concentrations.

Results. Fasting plasma insulin was associated with the triglyceride response to the test meal, independently of body mass index, waist-to-hip circumference ratio, blood glucose and the insulin effect on fasting plasma triglycerides. Exaggerated and prolonged postprandial lipaemia in subjects in the upper quartile of the plasma insulin distribution was largely accounted for by large (Svedberg flotation rate $>60$ )
\end{abstract}

very low density lipoproteins and chylomicron remnants. Insulin relations to large postprandial triglyceride-rich lipoproteins exclusively reflected the association between plasma insulin and the fasting plasma concentrations of these lipoprotein species, whereas plasma insulin and late postprandial plasma concentrations of small (Svedberg flotation rate 20-60) chylomicron remnants were related, independently of insulin influences on fasting concentrations. Strong positive relations were found between the late increases in large triglyceride-rich lipoproteins and plasma non-esterified fatty acid concentrations after $6 \mathrm{~h}$.

Conclusion/interpretation. The degree of insulin sensitivity is a major determinant of postprandial lipaemia in healthy middle-aged men and could add to the regulation of the basal production of large triglyceride-rich lipoproteins. [Diabetologia (2000) 43: 185-193]

Keywords Insulin, proinsulin, glucose, triglyceriderich lipoproteins, postprandial lipaemia.
Received: 16 August 1999 and in revised form: 22 October 1999

Corresponding author: Dr. S. Boquist, King Gustaf V Research Institute, Karolinska Hospital, S-171 76 Stockholm, Sweden Abbreviations: ANCOVA, Analysis of covariance; apo, apolipoprotein; CETP, cholesteryl ester transfer protein; E\%, energy \%; IAUC, incremental area under the curve; IDL, intermediate density lipoprotein; LPL, lipoprotein lipase; Sf, Svedberg flotation rate; TRL, triglyceride-rich lipoprotein; WHR, waistto-hip circumference ratio.
A cluster of risk factors for Type II (non-insulin-dependent) diabetes mellitus and cardiovascular disease, called the metabolic syndrome, is a common feature of otherwise healthy people living in industrialised countries. Increased visceral adipose tissue depots, insulin resistance with ensuing hyperinsulinaemia and dyslipoproteinaemia including hypertriglyceridaemia, a low HDL cholesterol concentration and a preponderance of small, dense LDL particles are central components of this syndrome [1]. The links between glucose-insulin homeostasis and lipoprotein metabolism have received particular atten- 
tion in the context of the metabolic syndrome. Relations between the fasting plasma insulin concentration, an adequate measure of insulin sensitivity in non-diabetic people [2] and fasting plasma lipoprotein concentrations have been established in many population-based studies in diverse ethnic groups [3]. The fasting plasma insulin concentration correlates positively with whole plasma and VLDL triglycerides and negatively with HDL cholesterol even in multivariate analyses controlled for important confounders such as age, sex, glucose tolerance, obesity and body fat distribution. Population-based studies have also reported associations between fasting plasma insulin and lipoprotein subclasses such as the inverse relations to the LDL peak particle size [4] and the $\mathrm{HDL}_{2}$ cholesterol concentration [5]. Furthermore, the plasma insulin concentration has proved to be related to the production of large VLDL particles with a Svedberg flotation (Sf) rate of 60 to 400 in experimental studies in humans using a stable isotope technique [6].

Enhanced and prolonged postprandial lipaemia has been related to coronary and carotid atherosclerosis and to the clinical manifestations of CHD [7-9]. The knowledge of how postprandial lipaemia is regulated is poor, although many factors are known to influence the lipaemic response, i.e. fasting plasma triglycerides, glucose tolerance, age, sex, diet, obesity, body fat distribution and apolipoprotein (apo) E genotype $[10,11]$. Little is known about the relations between plasma insulin concentrations and postprandial lipaemia and population-based studies in this area are still lacking. Inappropriate release of NEFA into the circulation in the postprandial state is, however, a hallmark of the metabolic syndrome and is likely both to reduce the sensitivity of glucose metabolism to insulin and to enhance postprandial lipaemia [12, 13]. In accordance with this, small studies have suggested possible roles of insulin resistance and hyperinsulinaemia as determinants of the postprandial plasma concentrations of triglyceride-rich lipoproteins (TRLs) in non-diabetic people $[14,15]$. Whether TRLs of intestinal origin are partly or entirely responsible for the relation between fasting plasma insulin concentration and postprandial lipaemia [15] is also under debate.

In this study, the relations of the fasting plasma insulin concentration to alimentary lipaemia and postprandial plasma concentrations of NEFA and TRLs were investigated in a population-based sample of healthy, 50-year-old men with an apo E3/3 genotype. The relations with obesity, body fat distribution and blood glucose and plasma proinsulin concentrations were also examined. Postprandial plasma triglycerides and the content of apo B-100 (reflecting VLDL and VLDL remnant particle numbers) and apo B-48 (reflecting chylomicron and chylomicron remnant particle numbers) in subfractions of TRLs were mea- sured to evaluate the lipid and lipoprotein responses to a mixed meal.

\section{Subjects and methods}

Subjects and study design. A total of 99 subjects, recruited from a population survey comprising 412 50-year-old Caucasian men living in the northern parts of the county of Stockholm, participated in the study. The original cohort was randomly selected from a registry comprising all permanent residents. Inclusion criteria for the present study, in addition to male sex and age of 50 years, were North European or North American descent and the presence of an apo E3/E3 genotype. Exclusion criteria were chronic disease of any kind, a history of CHD or arterial thromboembolic disease, familial hypercholesterolaemia, BMI more than $32 \mathrm{~kg} / \mathrm{m}^{2}$, alcohol abuse or psychiatric disorders that would interfere with compliance and participation in other ongoing studies. Of 412 men originally invited to participate in the programme, 324 (79\%) accepted. They then underwent a screening examination comprising blood sampling for fasting plasma lipoproteins, blood glucose, routine tests for liver, renal and thyroid functions and DNA isolation. Participants were also interviewed by a nurse who completed a questionnaire about medical history and current medical status, medication, smoking habits and alcohol consumption. Weight, height and waist and hip circumferences were measured, as was blood pressure, the latter after a 5-min rest in the supine position.

Screenees who were homozygous for the apo E3 allele and without any exclusion criteria were subsequently asked to return for an oral fat tolerance test, the protocol and composition of the mixed meal of which has been described previously [16]. The total energy content of the meal was $1000 \mathrm{kcal}$ with 60.2 energy \% (E\%) from fat (soybean oil), $13.3 \mathrm{E} \%$ from protein and $26.5 \mathrm{E} \%$ from carbohydrate. Blood samples were drawn after an overnight fast and then every hour for $6 \mathrm{~h}$ after ingestion of the test meal. In the fasting samples, blood glucose, plasma insulin, proinsulin and NEFA, cholesterol and triglycerides in plasma and major lipoprotein fractions and the apo B-48 and apo B-100 contents in Svedberg flotation rate (Sf) over 400, Sf 60-400, Sf 20-60 and Sf 12-20 lipoprotein fractions were determined. Plasma triglycerides were measured in all postprandial samples, whereas apo B-48 and apo B-100 were measured in subfractions of TRLs in the 3-h and 6-h samples. In addition, plasma NEFA were determined in the 3 -h and 6-h samples.

The study was approved by the ethics committee of the Karolinska Hospital. All subjects gave their informed consent to participation.

Lipoprotein determinations and other assays. Fasting plasma concentrations of cholesterol and triglycerides in VLDL, LDL and HDL were determined by a combination of preparative ultracentrifugation, precipitation of apo B-containing lipoproteins and lipid analyses [17]. Triglyceride-rich lipoproteins were subfractionated by cumulative density gradient ultracentrifugation [18]. Consecutive runs calculated to float Sf over 400, Sf 60-400 and Sf 20-60 particles were made and the Sf 12-20 fraction was recovered by slicing the tube after the Sf 20-60 lipoproteins had been aspirated. All Sf over 60 particles are referred to as large TRLs and Sf 20-60 particles as small TRLs.

The apo B-48 and apo B-100 concentrations in each TRL fraction were determined by analytical SDS-PAGE [19]. Gels were stained by Coomassie G-250 (Serva, Heidelberg, Germa- 
Table 1. Characteristics of the study cohort according to quartiles of fasting plasma insulin concentration

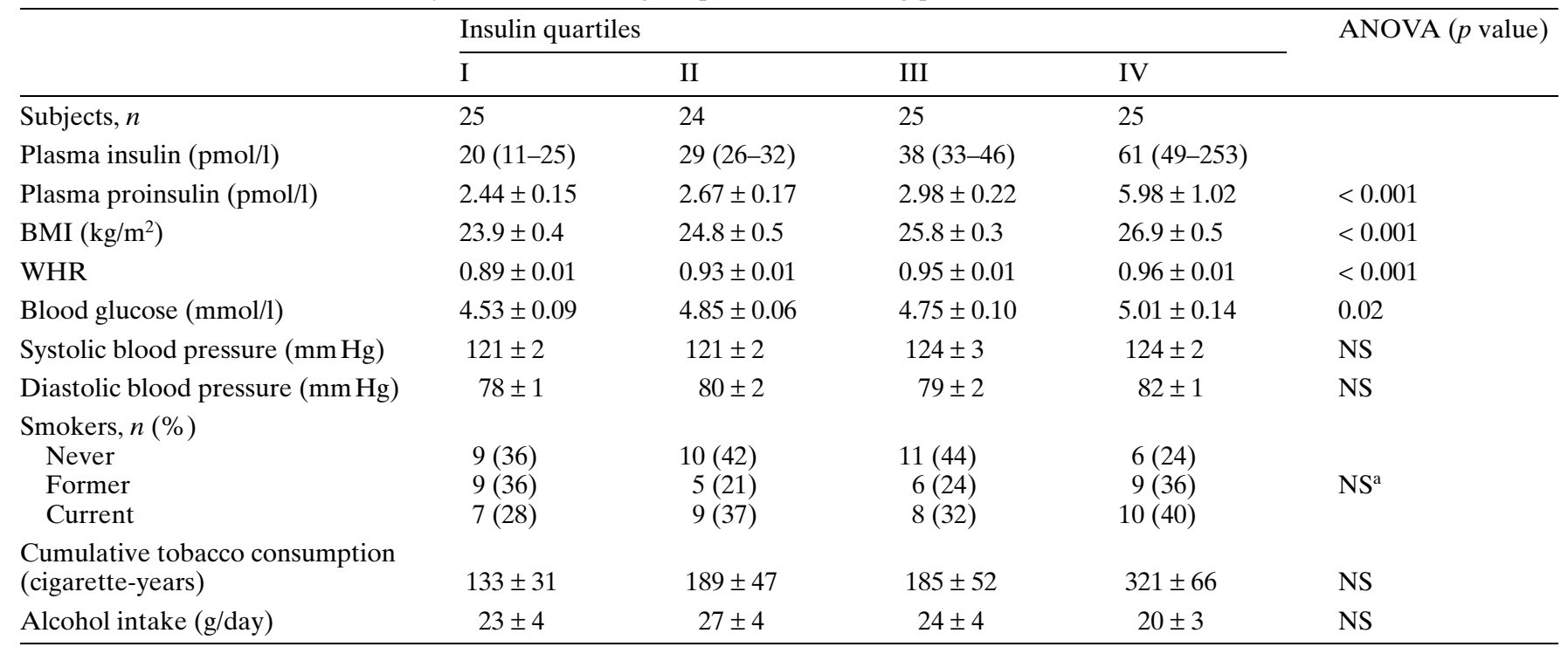

Values are means \pm SEM, except for plasma insulin (median and range). Insulin quartiles I-IV indicate lower-upper insulin quartiles. ${ }^{\text {a }}$ Chi-squared test

ny) and then scanned using a laser scanner connected to a personal computer, which was equipped with software providing automatic integration of areas under the scanning curves (Gelscan XL, Pharmacia, Stockholm, Sweden). Using the present methodology, the limit for detection of apo B-48 and apo B100 is $0.02 \mathrm{mg} / \mathrm{l}$ plasma concentration. Plasma NEFA were measured by an enzymatic colorimetric method (Wako Chemicals, Neuss, Germany).

A polymerase chain reaction technique was used to determine apo E genotype [20]. Glucose was measured in whole blood by a glucose oxidase method. Plasma insulin and proinsulin were measured by commercial ELISAs based on pairs of mouse monoclonal antibodies (DAKO Diagnostics, Cambridgeshire, UK). The cross-reactivity between insulin and proinsulin is $0.3 \%$ in the insulin assay. Within-assay and between-assay coefficients of variation for insulin determinations were $5.3 \%$ and $6.2 \%$, respectively. The detection limit for insulin is $3 \mathrm{pmol} / \mathrm{l}$.

Calculations and statistical analyses. Data are given as means \pm SEM. The incremental area under the curve (IAUC) of plasma triglycerides (determined by the trapezoid method) was used as a measure of the postprandial triglyceride response. All statistical analyses were conducted using the JMP statistical package (SAS Institute, Cary, N.C., USA). The individual values of skewed variables were log normalized before statistical tests. Relations of plasma insulin and NEFA to fasting and postprandial lipoproteins were first evaluated by ANOVA (insulin and NEFA quartiles used as categorical variables) and then by analysis of covariance (ANCOVA) (insulin and NEFA quartiles used as categorical variables), with adjustment for BMI, waist-to-hip circumference ratio (WHR) and blood glucose. Fasting plasma triglycerides or the fasting plasma concentration of the relevant TRL or of NEFA were also taken into account when analysing postprandial data. Relations of plasma proinsulin to fasting and postprandial lipoproteins were evaluated by ANCOVA (proinsulin used as a categorical variable), with adjustment for BMI, WHR and blood glucose. A $p$ value of less than 0.05 was considered as indicative of a statistically significant relation.

\section{Results}

Characteristics of the study group. The majority of the participants were normotensive, not obese and had normal fasting blood glucose and lipid values. Most of them also had a normal fasting plasma insulin concentration (only 15\% had an insulin concentration greater than $60 \mathrm{pmol} / \mathrm{l})$. Plasma insulin quartiles were strongly related to BMI, WHR, and plasma proinsulin concentrations, whereas the association with the blood glucose concentration was barely significant (Table 1).

Relations between plasma insulin and fasting lipoproteins. The plasma insulin concentration was significantly related to fasting VLDL cholesterol and triglycerides and to HDL cholesterol, even after adjustment for BMI, WHR and blood glucose, when subjects were grouped according to quartiles of the fasting plasma insulin concentration (Table 2). The independent association of insulin with VLDL lipids was mainly accounted for by large VLDLs (Sf $>60$ apo B$100)$. Fasting plasma insulin was also independently related to the fasting plasma concentration of large and small chylomicron remnants (Sf $>60$ apo B-48 and Sf 20-60 B-48, respectively). The intermediate density lipoprotein (IDL) (Sf 12-20 apo B-100) concentration was also independently related to plasma insulin quartiles. In contrast, the significant univariate association between plasma insulin quartiles and LDL triglycerides disappeared after adjustment for BMI, WHR and blood glucose. Furthermore, there were no statistically significant relations between the fasting plasma insulin concentration and fasting plasma concentrations of small VLDLs (Sf 20-60 apo B-100). 
Table 2. Relations between quartiles of fasting plasma insulin concentration and fasting plasma lipid and lipoprotein concentrations

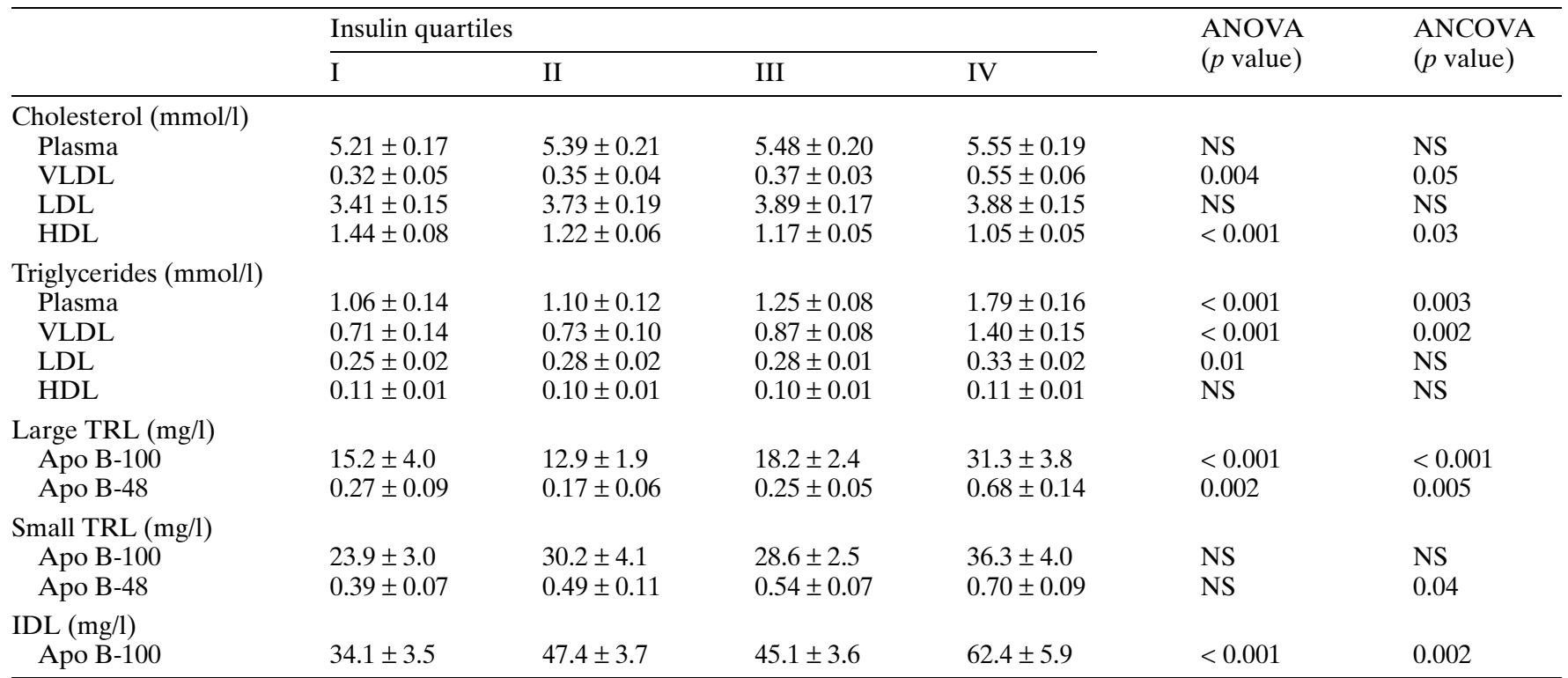

Values are means \pm SEM. Insulin quartiles I-IV indicate lower-upper insulin quartiles; ANCOVA indicates analysis of covariance adjusted for BMI, WHR and blood glucose concentra- tion; large TRL, Sf $>60$ lipoprotein fraction; small TRL, Sf 20-60 lipoprotein fraction; IDL, intermediate density lipoprotein (Sf 12-20 lipoprotein fraction)

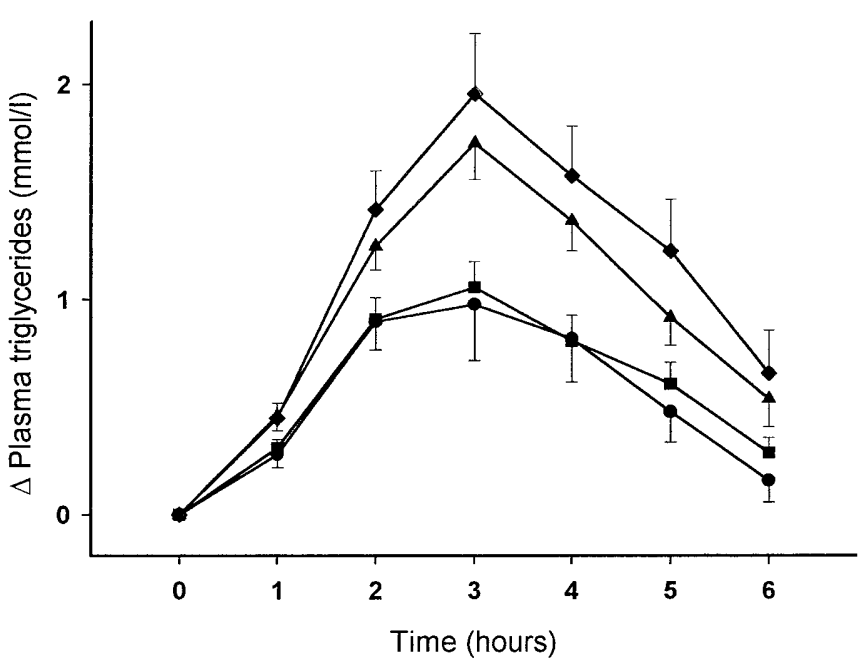

Fig. 1. Postprandial triglyceride response to a mixed meal in healthy 50-year-old men who were grouped according to quartiles of the fasting plasma insulin concentration. Values are least squares means \pm SEM. $\Delta$ indicates changes with respect to baseline; QI-QIV lower to upper insulin quartiles. The integrated triglyceride response was evaluated as the incremental area under the postprandial curve (IAUC) and found to differ significantly according to insulin quartile $(p=0.001$ in ANOVA, $p=0.02$ in ANCOVA with adjustment for fasting plasma triglycerides and $p=0.02$ in ANCOVA with adjustment for fasting plasma triglycerides, BMI, WHR and fasting blood glucose). - - , QI; - - , QII; - -, QIII; $\bullet$, QIV
The corresponding relations of the plasma proinsulin concentration were also analysed with adjustment for BMI, WHR and blood glucose. No association was found between quartiles of plasma proinsulin and VLDL cholesterol, HDL cholesterol and IDL using these covariates. Otherwise, the same independent associations with plasma lipid and lipoprotein concentrations were present for plasma proinsulin (data not shown) and plasma insulin (Table 2). Conversely, BMI, WHR, and blood glucose were not related to fasting plasma lipoproteins, when plasma insulin concentrations were taken into account (data not shown).

Relations between plasma insulin and postprandial lipids and lipoproteins. The postprandial plasma triglyceride response to the mixed meal, illustrated in Fig. 1 and measured as the IAUC for triglycerides, was significantly related to the quartile distribution of the fasting plasma insulin concentration, independently of fasting plasma triglyceride concentration, BMI, WHR and blood glucose concentration (Table 3).

The postprandial plasma concentrations of individual TRLs at 3 and $6 \mathrm{~h}$ after intake of the test meal in subjects grouped according to plasma insulin quartile are shown in Table 3. The postprandial plasma concentrations of large VLDLs and large chylomicron remnants were associated with the fasting plasma insulin concentration. These relations were mainly accounted for by the subjects in the upper insulin quartile. Covariance analyses showed, however, that 
Table 3. Relations between quartiles of fasting plasma insulin concentration and fasting and postprandial plasma concentrations of triglyceride-rich lipoproteins and NEFA

\begin{tabular}{|c|c|c|c|c|c|c|c|}
\hline & \multicolumn{4}{|c|}{ Insulin quartiles } & \multirow{2}{*}{$\begin{array}{l}\text { ANOVA } \\
(p \text { value })\end{array}$} & \multirow{2}{*}{$\begin{array}{l}\text { ANCOVA } 1 \\
(p \text { value })\end{array}$} & \multirow{2}{*}{$\begin{array}{l}\text { ANCOVA } 2 \\
(p \text { value })\end{array}$} \\
\hline & I & II & III & IV & & & \\
\hline $\begin{array}{l}\text { IAUC triglycerides } \\
(\mathrm{mmol} / \mathrm{lxh})\end{array}$ & $3.5 \pm 0.7$ & $3.9 \pm 0.4$ & $6.0 \pm 0.6$ & $7.0 \pm 0.9$ & 0.001 & 0.02 & 0.02 \\
\hline \multicolumn{8}{|l|}{ Large TRL (mg/l) } \\
\hline \multicolumn{8}{|l|}{ Apo B-100 } \\
\hline Oh & $15.2 \pm 4.0$ & $12.9 \pm 1.9$ & $18.2 \pm 2.4$ & $31.3 \pm 3.8$ & $<0.001$ & - & - \\
\hline \multicolumn{8}{|l|}{ Apo B-48 } \\
\hline $\mathrm{Oh}$ & $0.27 \pm 0.09$ & $0.17 \pm 0.06$ & $0.25 \pm 0.05$ & $0.68 \pm 0.14$ & 0.002 & - & - \\
\hline $3 h$ & $1.19 \pm 0.24$ & $1.28 \pm 0.20$ & $1.66 \pm 0.16$ & $2.27 \pm 0.27$ & 0.003 & NS & NS \\
\hline $6 \mathrm{~h}$ & $0.67 \pm 0.22$ & $0.55 \pm 0.12$ & $0.87 \pm 0.15$ & $1.47 \pm 0.25$ & 0.003 & NS & NS \\
\hline \multicolumn{8}{|l|}{ Small TRL (mg/l) } \\
\hline \multicolumn{8}{|l|}{ Apo B-100 } \\
\hline $3 h$ & $0.57 \pm 0.08$ & $0.67 \pm 0.10$ & $0.78 \pm 0.06$ & $0.94 \pm 0.09$ & 0.01 & NS & NS \\
\hline $6 \mathrm{~h}$ & $0.32 \pm 0.05$ & $0.47 \pm 0.09$ & $0.51 \pm 0.07$ & $0.72 \pm 0.06$ & 0.002 & 0.05 & 0.04 \\
\hline \multicolumn{8}{|c|}{ Plasma NEFA (mmol/l) } \\
\hline Oh & $0.36 \pm 0.03$ & $0.41 \pm 0.03$ & $0.35 \pm 0.02$ & $0.34 \pm 0.03$ & NS & - & - \\
\hline $3 h$ & $0.33 \pm 0.02$ & $0.37 \pm 0.03$ & $0.37 \pm 0.03$ & $0.34 \pm 0.02$ & NS & NS & NS \\
\hline $6 \mathrm{~h}$ & $0.27 \pm 0.02$ & $0.32 \pm 0.02$ & $0.29 \pm 0.02$ & $0.35 \pm 0.02$ & NS & NS & NS \\
\hline
\end{tabular}

Values are means \pm SEM. Insulin quartiles I-IV indicate lower-upper insulin quartiles; ANCOVA 1 indicates analysis of covariance using the corresponding fasting plasma triglyceride, lipoprotein or NEFA concentration as covariate; ANCOVA 2 used the same covariate as in ANCOVA 1 along with BMI, WHR and blood glucose concentration; large TRL, Sf $>60$ lipoprotein fraction; small TRL, Sf 20-60 lipoprotein fraction the differences in postprandial plasma concentrations of large TRLs according to fasting insulin concentration depended entirely on differences present already in the fasting state and were not related to the postprandial response of the large TRLs. The postprandial plasma concentrations of the small chylomicron remnants were also significantly higher in subjects in the upper insulin quartile (Table 3 ). In contrast to the large TRLs, the relations of the postprandial small chylomicron remnants to fasting plasma insulin concentration seen at $6 \mathrm{~h}$ after intake of the test meal was independent of their fasting concentration (and of BMI, WHR and blood glucose). Postprandial plasma concentrations of small VLDLs, on the other hand, were not related to the fasting plasma insulin concentration.

The quartile distribution of the fasting plasma proinsulin concentration showed similar associations with the postprandial plasma concentrations of large VLDLs and large chylomicron remnants as did plasma insulin quartiles (data not shown), i.e. differences in postprandial plasma concentrations of large TRLs according to fasting proinsulin concentration depended entirely on differences present already in the fasting state and were not related to the postprandial responses of the large TRLs. In contrast to plasma insulin, no relation was, however, found between the plas- ma proinsulin concentration and the late postprandial plasma concentration of small chylomicron remnants that was independent of the fasting plasma concentration of small chylomicron remnants and of BMI, WHR and blood glucose.

The late postprandial plasma concentrations of NEFA tended to be higher in subjects in the upper insulin quartile (Table 3, $p=0.06$ in ANOVA for the plasma concentrations at $6 \mathrm{~h}$ ). A corresponding, considerably stronger relation was observed between plasma proinsulin quartiles and the late postprandial plasma concentration of NEFA [proinsulin quartile I(QI): $0.28 \pm 0.02$ (means \pm SEM); QII: $0.28 \pm 0.02$; QIII: $\quad 0.30 \pm 0.02 ;$ and $\mathrm{QIV}: \quad 0.37 \pm 0.03 \mathrm{mmol} / 1$ NEFA, $p=0.008$ (after adjustment for fasting NEFA, BMI, WHR and blood glucose)].

Of note, none of the potential confounding factors (BMI, WHR and blood glucose) was independently related to postprandial lipoproteins, when plasma insulin or proinsulin concentrations were taken into account (data not shown).

Relations between postprandial plasma NEFA and postprandial TRLs. Because the plasma concentrations of NEFA tended to be higher in subjects in the upper insulin quartile at $6 \mathrm{~h}$ after intake of the test meal, suggesting that insulin-mediated suppression 


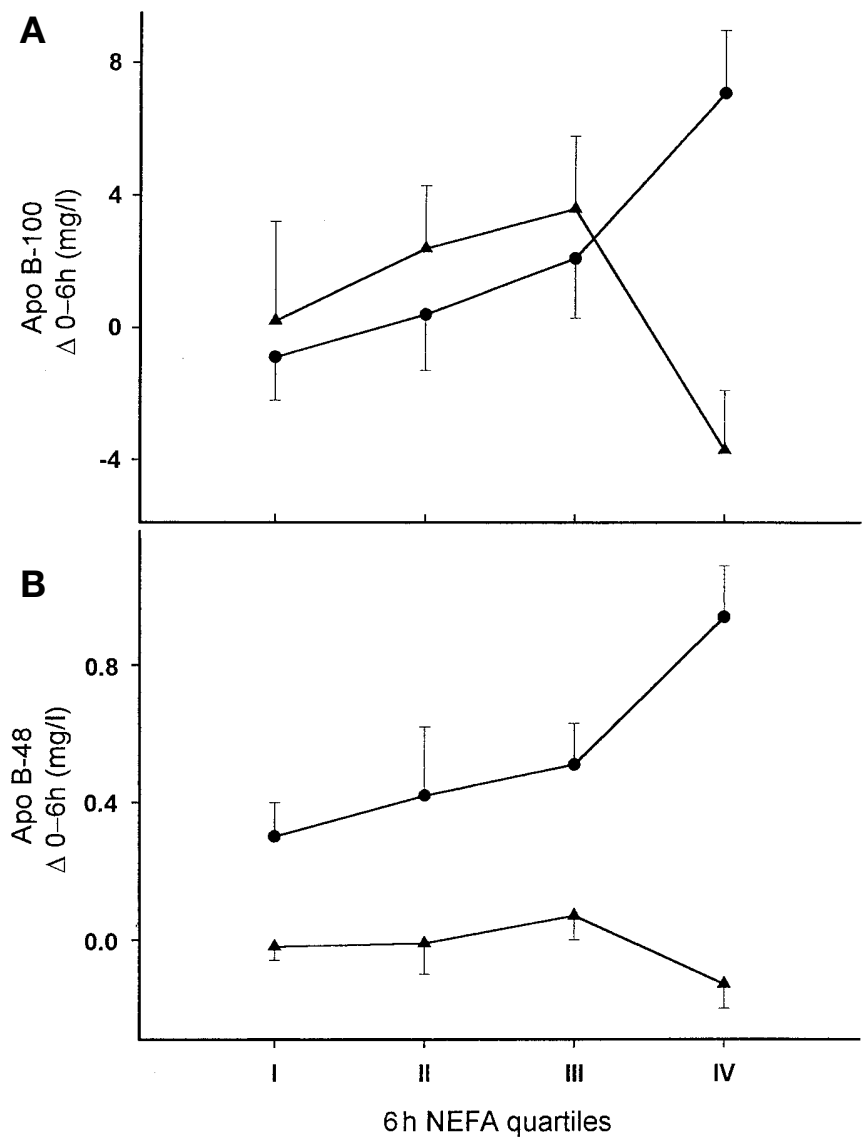

Fig. 2A, B. Postprandial responses of large $(\mathrm{Sf}>60, \mathbf{0})$ and small (Sf 20-60, $\boldsymbol{\Delta}$ ) triglyceride-rich lipoproteins to a mixed meal in healthy 50-year-old men who were grouped according to quartiles of the plasma NEFA concentration at $6 \mathrm{~h}$ after intake of the test meal. Values are least squares mean \pm SEM. Sf $>60$ apo B-100 represents large VLDLs; Sf 20-60 apo B100 , small VLDLs; Sf $>60$ apo B-48, large chylomicron remnants and Sf 20-60 apo B-48, small chylomicron remnants. $\Delta 0-6 \mathrm{~h}$ Indicates change between baseline fasting value and $6 \mathrm{~h}$. A A statistically highly significant, graded, positive relation was found between the increase in large VLDL from baseline to 6-h ( $\Delta 0-6 \mathrm{~h} \mathrm{Sf}>60$ apo B-100) and plasma NEFA concentrations at $6 \mathrm{~h}(p=0.02$ in ANCOVA with adjustment for plasma insulin, BMI, WHR and blood glucose). The corresponding relation for small VLDLs was not significant $(p=0.12)$. B A graded positive relation also existed between the increase in large chylomicron remnants from baseline to $6 \mathrm{~h}(\Delta 0-6 \mathrm{~h} \mathrm{Sf}>60$ apo B-48) and plasma NEFA concentrations at $6 \mathrm{~h}(p=0.03$ in ANCOVA with adjustment for plasma insulin, BMI, WHR and blood glucose). In contrast, the increase in small chylomicron remnants from baseline to $6 \mathrm{~h}$ was not related to plasma NEFA concentrations at $6 \mathrm{~h}$ $(p=0.17)$

of NEFA was impaired in these subjects, NEFA relations to responses of individual postprandial lipoproteins were subsequently examined to further explain the role of insulin/insulin sensitivity in postprandial lipoprotein metabolism. A statistically highly significant, graded, positive relation was found between the increase in large VLDL from baseline to $6 \mathrm{~h}$ $(\Delta 0-6 \mathrm{~h}$ Sf $>60$ apo B-100) and plasma NEFA con- centrations at $6 \mathrm{~h}$ (Fig. 2). This relation was independent of the fasting plasma insulin concentration ( $p=0.02$ in ANCOVA with adjustment for plasma insulin, BMI, WHR and blood glucose). A similar relation existed between the increase in large chylomicron remnants from baseline to $6 \mathrm{~h}(\Delta 0-6 \mathrm{~h} \mathrm{Sf}>60$ apo B-48) and plasma NEFA concentrations at $6 \mathrm{~h}$ (Fig. 2), which was also independent of the fasting plasma insulin concentration ( $p=0.03$ in ANCOVA with adjustment for plasma insulin, BMI, WHR and blood glucose). In contrast, NEFA concentrations at $6 \mathrm{~h}$ after intake of the test meal were not related to the responses of the small TRL species (Fig. 2).

\section{Discussion}

The primary objective of our study was to investigate the relations of the fasting plasma insulin concentration, an adequate surrogate measure of degree of insulin sensitivity, to postprandial lipaemia and plasma concentrations of individual postprandial TRLs in a population-based group of largely non-obese, healthy middle-aged men. The main finding was a fairly strong positive association between plasma insulin and the triglyceride response to the test meal that was independent of BMI, WHR, blood glucose and the insulin effect on fasting plasma triglycerides. Furthermore, the plasma insulin concentration was strongly related to the postprandial plasma concentrations of large VLDLs and large chylomicron remnants. These relations exclusively reflected, however, the association between plasma insulin and the fasting plasma concentrations of these lipoprotein species, whereas the postprandial responses of the large TRLs were not related to the fasting plasma insulin concentration. In contrast, the association between plasma insulin and the late postprandial plasma concentrations of small chylomicron remnants was not entirely dependent on the corresponding association of plasma insulin with the fasting plasma concentrations of this remnant species. The corollary is that insulin sensitivity could add to the regulation of the basal production of large TRLs, whereas other mechanisms determine the postprandial responses of these lipoproteins. The postprandial responses of small chylomicron remnants, on the other hand, are to some extent influenced by insulin sensitivity.

In the fasting state, plasma insulin was independently related to the VLDL triglyceride and HDL cholesterol concentrations as well as to the plasma concentrations of large (Sf > 60) VLDL, large chylomicron remnant and IDL particles. The associations between plasma insulin and fasting VLDL triglycerides (positive) and HDL cholesterol (inverse) have been shown repeatedly before [3]. The increased VLDL triglyceride concentration seen in the highest insulin quartile was paralleled by statistically signifi- 
cantly higher fasting plasma concentrations of large TRLs. Insulin has been shown to regulate the production rate of large, but not small, VLDL particles in humans [6]. It is reasonable to believe that subjects in the highest insulin quartile in our cohort are to some extent insulin resistant and, as a consequence, the suppressive effect of insulin on the production of large VLDLs is reduced. The link between insulin concentration and postprandial chylomicron response might, on the other hand, reflect the ensuing increased competition for lipoprotein lipase (LPL) between VLDL and chylomicrons.

The late postprandial plasma concentrations of NEFA, which were not adequately suppressed in the least insulin-sensitive subjects (those in the upper insulin quartile), were positively related to a prolonged postprandial increase in large VLDLs and large chylomicron remnants. The link between perturbed postprandial metabolism of NEFA and large TRLs was not entirely dependent on concomitant insulin resistance or impaired glucose tolerance or either of them, because the relation of the NEFA concentration at $6 \mathrm{~h}$ after intake of the test meal to increases in large TRLs between baseline and $6 \mathrm{~h}$ remained statistically significant even after adjustment for fasting plasma insulin concentration, BMI, WHR and blood glucose. Thus, the NEFA effects on the postprandial metabolism of large TRLs appear to be not solely a consequence of the degree of insulin sensitivity of the individual person. The rate of hepatic VLDL secretion is strongly dependent on the NEFA supply [21]. Failure to suppress the NEFA supply to the liver in the postprandial state could lead to sustained VLDL production and impaired clearance of postprandial TRLs through competition of the large VLDLs with chylomicrons and large chylomicron remnants for hydrolysis by LPL. In addition, local accumulation of NEFA could lead to inactivation of LPL by dissociating the enzyme from its endothelial binding site [22, 23].

We found that fasting plasma insulin is independently associated with the postprandial triglyceride response. This is in agreement with some $[15,24]$ but not all previous studies $[14,25]$. There was also an independent positive relation between plasma insulin and the late postprandial particle concentration of small chylomicron remnants. The latter observation extends the previous finding of a positive correlation between day-long insulin and postprandial retinyl palmitate responses [15]. Enhanced alimentary lipaemia and increased postprandial plasma concentrations of TRLs have important clinical and metabolic consequences. Peak and late postprandial triglyceride concentrations have been associated with coronary atherosclerosis [7, 26, 27] and early carotid atherosclerosis [8, 28]. Furthermore, postprandial retinyl palmitate concentrations, reflecting postprandial concentrations of chylomicrons and chylomicron remnants, are associated with CHD in case-control studies $[29,30]$ and postprandial plasma concentrations of small chylomicron remnants are related to progression of coronary lesions as determined by sequential coronary angiographies [31].

The triglyceride response to the test meal is an independent predictor of common carotid artery intima-media thickness (IMT) in the present cohort, as measured by B-mode ultrasound examination [9]. The main finding of the present report of a fairly strong positive association between plasma insulin and degree of postprandial triglyceridaemia, that is independent of BMI, WHR, blood glucose and the insulin effect on fasting plasma triglycerides, sheds new light on the relation between alimentary lipaemia and early atherosclerosis. The insulin relation with postprandial triglyceridaemia suggests that a person's plasma insulin concentration/insulin sensitivity is a basic pathogenic factor in atherogenesis, the effect of which is at least partly mediated by postprandial TRLs.

The exaggerated postprandial lipaemia also has consequences for the particle composition of all major plasma lipoproteins. These effects are mediated by the cholesteryl ester transfer protein (CETP), which catalyses the transfer of cholesteryl esters from HDL and LDL to chylomicrons, VLDL and IDL and the reciprocal transfer of triglycerides [32]. Triglyceride-enriched LDL and HDL particles are good substrates for hepatic lipase and the result is smaller and denser LDL and HDL particles, constituting a more atherogenic lipoprotein phenotype. It is notable in this context that increased NEFA concentrations, present postprandially in the more insulin-resistant subjects in this study, potentiate the activity of CETP [33] and thus promote the atherogenic lipoprotein phenotype.

Recently, the postprandial responses of TRLs were compared between two groups of men matched for fat mass but with either high or low visceral adiposity as assessed by computed tomography [34]. An increased response of medium and small TRL triglyceride and higher plasma concentrations of these particles and TRL triglyceride were observed at $8 \mathrm{~h}$ after intake of the test meal in the group with high amounts of visceral adipose tissue. Furthermore, the increase in retinyl palmitate contained in total, large and medium TRLs was higher in the men with greater visceral adiposity as were the IAUCs for insulin and NEFA. In contrast, there was no difference in the fasting plasma triglyceride concentration. We did not find any independent relations between BMI and WHR on the one hand and fasting or postprandial TRLs on the other, insulin being the main determinant of postprandial lipaemia independently of BMI or fat distribution. This discrepancy could be explained by smaller differences in visceral adipose tissue depots amongst the healthy 50 -year-old men participating in the study and also by the different tech- 
niques used to assess visceral obesity. Clearly, insulin was a more important determinant of postprandial lipaemia in our cohort.

The current study in several ways provides further insight into the relations between glucose-insulin homeostasis and metabolism of TRLs. Compared to earlier studies, our group of healthy 50-year-old men with an apo E3/3 genotype is more homogeneous and population-based, which reduces the influence of confounders. We also used a technique, SDSPAGE of subfractions of TRLs, that allowed us to quantify TRLs of different origin and particle size more accurately than the combination of TRL triglyceride and retinyl palmitate determinations.

In summary, our study shows a strong association between basal plasma insulin and postprandial triglyceridaemia, independently of abdominal obesity. The exaggerated and prolonged triglyceride response to a standard meal in hyperinsulinaemic subjects is accounted for by large TRLs. Furthermore, the plasma insulin concentration correlates with the particle concentration of large VLDLs in the fasting state, a finding which might reflect increased basal production of large VLDL in insulin-resistant people without manifest Type II diabetes. The NEFA effects on the postprandial metabolism of large TRLs appear not to be solely a consequence of the degree of insulin sensitivity of the individual person.

Acknowledgements. The authors are grateful to Dr. B. V.Howard for valuable comments on the manuscript. This study was supported by grants from the Swedish Heart-Lung Foundation, the Swedish Medical Research Council (8691), the Novo Nordisk Foundation, the Marianne and Marcus Wallenberg Foundation and the Karolinska Institute. Dr. Ruotolo was supported by a research fellowship from the Swedish Institute, San Raffaele Scientific Institute, Milan, Italy.

\section{References}

1. Grundy SM (1998) Hypertriglyceridemia, atherogenic dyslipidemia, and the metabolic syndrome. Am J Cardiol 81(4A): 18B-25B

2. Laakso M, Sarlund H, Mykkänen L (1990) Insulin resistance is associated with lipid and lipoprotein abnormalities in subjects with varying degrees of glucose tolerance. Arterioscler Thromb 10: 223-228

3. Howard BV (1997) Insulin actions in vivo: insulin and lipoprotein metabolism. In: Alberti KG, Zimmet P, DeFronzo RA, Keen H (eds). International textbook of diabetes mellitus. 2nd edn. Wiley, Chichester, pp 531-539

4. Haffner SM, Mykkänen L, Valdez RA, Paidi M, Stern MP, Howard BV (1993) LDL size and subclass pattern in a biethnic population. Arterioscler Thromb 13: 1623-1630

5. Ostlund RE, Staten M, Kohrt WM, Schultz J, Malley M (1990) The ratio of waist-to-hip circumference, plasma insulin level, and glucose intolerance as independent predictors of the $\mathrm{HDL}_{2}$ cholesterol level in older adults. N Engl J Med 322: 229-234

6. Malmström R, Packard CJ, Watson TD et al. (1997) Metabolic basis of hypotriglyceridemic effects of insulin in normal men. Arterioscler Thromb Vasc Biol 17: 1454-1464
7. Patsch JR, Miesenböck G, Hopferwieser Tet al. (1992) Relation of triglyceride metabolism and coronary artery disease. Arterioscler Thromb 12: 1336-1345

8. Ryu JE, Howard G, Craven TE, Bond MG, Hagaman AP, Crouse III JR (1992) Postprandial triglyceridemia and carotid atherosclerosis in middle-aged subjects. Stroke 23: 823-828

9. Boquist S, Ruotolo G, Tang R et al. (1999) Alimentary lipemia, postprandial triglyceride-rich lipoproteins, and common carotid intima-media thickness in healthy, middle-aged men. Circulation 100: 723-728

10. Taskinen M-R (1995) Insulin resistance and lipoprotein metabolism. Curr Opin Lipidol 6: 153-160

11. Karpe F, Hamsten A (1995) Postprandial lipoprotein metabolism and atherosclerosis. Curr Opin Lipidol 6: 123-129

12. Frayn KN, Williams CM, Arner P (1996) Are increased plasma non-esterified fatty acid concentrations a risk marker for coronary heart disease and other chronic diseases? Clin Sci 90: (Colch) 243-253

13. Frayn KN (1998) Non-esterified fatty acid metabolism and postprandial lipaemia. Atherosclerosis 141:[Suppl]: S41-S46

14. Weintraub MS, Eisenberg S, Breslow JL (1987) Different pattern of postprandial lipoprotein metabolism in normal, type IIa, type III, and type IV hyperlipoproteinemic individuals. J Clin Invest 79: 1110-1119

15. Jeppesen J, Hollenbeck CB, Zhou MY et al. (1995) Relation between insulin resistance, hyperinsulinemia, postheparin plasma lipoprotein lipase activity, and postprandial lipemia. Arterioscler Thromb Vasc Biol 15: 320-324

16. Boquist S, Ruotolo G, Hellenius ML, Danell-Toverud K, Karpe F, Hamsten A (1998) Effects of a cardioselective beta-blocker on postprandial triglyceride-rich lipoproteins, low density lipoprotein particle size and glucose-insulin homeostasis in middle-aged men with modestly increased cardiovascular risk. Atherosclerosis 137: 391-400

17. Carlson K (1973) Lipoprotein fractionation. J Clin Pathol 5: $32-37$

18. Karpe F, Steiner G, Olivecrona T, Carlson LA, Hamsten A (1993) Metabolism of triglyceride-rich lipoproteins during alimentary lipemia. J Clin Invest 91: 748-758

19. Karpe F, Hamsten A (1994) Determination of apolipoproteins B-48 and B-100 in triglyceride-rich lipoproteins by sodium dodecyl sulfate polyacrylamide gel electrophoresis. J Lipid Res 35: 1311-1317

20. van den Maagdenberg AM, de Knijff P, Stalenhoef AF, Gevers Leuven JA, Havekes LM, Frants RR (1989) Apolipoprotein $\mathrm{E}^{*} 3$-Leiden allele results from a partial gene duplication in exon 4. Biochem Biophys Res Commun 165: $851-857$

21. Byrne CD, Brindle NP, Wang TW, Hales CN (1991) Interaction of non-esterified fatty acid and insulin in control of triacylglycerol secretion by HepG2 cells. Biochem J 280: 99-104

22. Saxena U, Witte LD, Goldberg IJ (1989) Release of endothelial cell lipoprotein lipase by plasma lipoproteins and free fatty acids. J Biol Chem 264: 4349-4355

23. Peterson J, Bihain BE, Bengtsson-Olivecrona G, Deckelbaum RJ, Charpentier Y, Olivecrona T (1990) Fatty acid control of lipoprotein lipase: a link between energy metabolism and lipid transport. Proc Natl Acad Sci USA 87:909-913

24. Schrezenmeir J, Keppler I, Fenselau S et al. (1993) The phenomenon of a high triglyceride response to an oral lipid load in healthy subjects and its link to the metabolic syndrome. Ann NY Acad Sci 683: 302-314

25. Byrne CD, Wareham NJ, Phillips DI, Hales CN, Martensz ND (1997) Is an exaggerated postprandial triglyceride re- 
sponse associated with the component features of the insulin resistance syndrome? Diabet Med 14: 942-950

26. Ginsberg HN, Jones J, Blaner WS et al. (1995) Association of postprandial triglyceride and retinyl palmitate responses with newly diagnosed exercise-induced myocardial ischemia in middle-aged men and women. Arterioscler Thromb Vasc Biol 15: 1829-1838

27. Simpson HS, Williamson CM, Olivecrona T et al. (1990) Postprandial lipemia, fenofibrate and coronary artery disease. Atherosclerosis 85: 193-202

28. Karpe F, de Faire U, Mercuri M, Bond M, Hellénius M-L, Hamsten A (1998) Magnitude of alimentary lipemia is related to intima-media thickness of the common carotid artery in middle-aged men. Atherosclerosis 141: 307-314

29. Weintraub MS, Grosskopf I, Rassin T et al. (1996) Clearance of chylomicron remnants in normolipidaemic patients with coronary artery disease: case control study over three years. BMJ 321: 935-939
30. Meyer E, Westerveld HT, de Ruyter-Meijstek FC et al. (1996) Abnormal postprandial apolipoprotein B-48 and triglyceride responses in normolipidemic women with greater than $70 \%$ stenotic coronary artery disease: a casecontrol study. Atherosclerosis 124: 221-235

31. Karpe F, Steiner G, Uffelman K, Olivecrona T, Hamsten A (1994) Postprandial lipoproteins and progression of coronary atherosclerosis. Atherosclerosis 106: 83-97

32. Tall A (1993) Plasma cholesteryl ester transfer protein. J Lipid Res 34: 1255-1274

33. Lagrost L, Florentin E, Guyard Dangremont Vet al. (1995) Evidence for nonesterified fatty acids as modulators of neutral lipid transfers in normolipidemic human plasma. Arterioscler Thromb Vasc Biol 15: 1388-1396

34. Couillard C, Bergeron N, Prud'homme D et al. (1998) Postprandial triglyceride response in visceral obesity in men. Diabetes 47: 953-960 